\title{
LEISTUNGSERBRINGER
}

\section{Vom Gestalter zum Getriebenen}

\author{
Ludger Lünenborg
}

Für die Träger der beruflichen Integration benachteiligter Jugendlicher und Erwachsener begann vor zehn Jahren der grundlegende Wandel in ihrer Arbeit: Maßnahmen zur beruflichen Bildung werden seither mittels Ausschreibungen vergeben. Seither ist ein vielfach ruinöser Preisverfall $\mathrm{zu}$ beobachten.

Wie viel und welche Art von Unterstützung braucht ein Abgänger einer Förderschule, um erfolgreich und dauerhaft seinen Platz in der Arbeitswelt zu finden? Das war eine der wichtigen Fragen, deren Beantwortung die gestaltende Arbeit eines Geschäftsführers eines Trägers der Jugendsozialarbeit und Jugendberufshilfe bestimmt hat. Es ging um Konzepte, Arbeitsprojekte und ihre Wirkungen.

Das war in den achtziger und Anfang der neunziger Jahre des vorigen Jahrhunderts so. Für uns als regionaler Träger der beruflichen Bildung endete diese Zeit vor etwas mehr als zehn Jahren mit der ersten Vergabe der »Ausbildungsbegleitenden Hilfen $(\mathrm{abH})$ « mittels Ausschreibung im Jahr 1997. Seit dem stehen eher Fragen im Vordergrund wie »Welcher Bereich in der Bewertungsmatrix ist mit dem höchsten Faktor versehen?« oder noch wichtiger: »Zu welchem Preis hat der Träger X, der seine Aufträge in den neuen Bundesländern verloren hat, im letzten Jahr in der benachbarten Arbeitsagentur bei der dortigen abH-Ausschreibung angeboten? « und »Was zahlt er seinen Mitarbeitern?«.

Damit verbunden ist eine grundlegende Aufgabenverschiebung. Sie zwingt Organisationen, die ideelle Ziele verfolgen - in unserem Fall die Förderung und Unterstützung von benachteiligten und beeinträchtigten Jugendlichen - in eine Organisationsentwicklung, die sich fast ausschließlich an der Bedienung der Nachfragen seitens der öffentlichen Auftraggeber orientiert.

\section{Der sogenannte Qualitätswettbewerb}

Bis zur Einführung des SGB II im Jahre 2005 waren für Unterstützungsleistungen im Bereich der beruflichen Integration von benachteiligten Jugendlichen die Arbeitsämter oder die Arbeitsagenturen die Auftraggeber, an wenigen Orten auch noch örtliche Jugend- oder Sozialämter. Während zu Beginn der Auftragsvergaben unter der VOL/A (Verdingungsordnung für Leistungen) noch die örtlichen Arbeitsämter eigenständig die Ausschreibungen durchführten, wurde mit der Restrukturierung der Arbeitsverwaltung (»Arbeitsamt 2000«) zu Beginn des Jahrtausends das »BA-Service-Haus « als zentrale Vergabestelle für Aufträge der Bundesagentur für Arbeit (BA) eingerich-

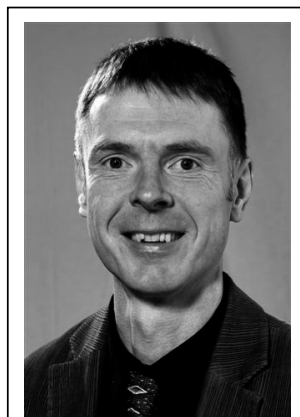

Ludger Lünenborg (48) ist seit 1986 in der beruflichen Bildung tätig, zunächst als Lehrer in der außerschulischen Berufsausbildung, seit 1993 in der Geschäftsführung der Organisation »Lernen fördern e. V. Kreisverband Steinfurt", einem regionalen Träger von Einrichtungen und Projekten zur beruflichen und gesellschaftlichen Integration benachteiligter Jugendlicher und Erwachsener. Seine Tätigkeitsschwerpunkte sind Angebotsentwicklung, Projektsteuerung und Personalentwicklung. E-Mail luenenborg@lernenfoerdern.de

tet. Damit hielt ein bis ins Detail formalisierter Vergabeprozess Einzug, der nicht nur Abgabefristen auf die Minute genau vorgibt, sondern auch die Seitenanzahl eines Angebotskonzeptes und die Reihenfolge der darzustellenden Konzeptbereiche exakt definiert. Abweichungen hiervon führen zum Ausschluss des Angebotes und damit schuf das Verfahren zu Beginn viele Opfer wegen fehlender Unterschriften oder zu umfangreicher Angebote.

Bei den ersten Vergabeverfahren wurden Losgrößen ausgeschrieben, die die Hälfte des Gebietes des Bundeslandes Nordrhein-Westfalen umfassten. Regionale Träger, die sich für ihr Gebiet beteiligen wollten, schlossen sich mit ihnen unbekannten Trägern aus anderen Regionen zu einer Bietergemeinschaft zusammen. Ich war an Angebotsbesprechungen beteiligt, wo circa 15 Trägervertreter versuchten, sich auf ein gemeinsames Konzept und noch viel schwieriger auf einen gemeinsamen Preis zu verständigen, ohne die Karten (= trägerinterne betriebswirtschaftliche Kalkulationen und trägerspezifische Angebotsbausteine) aufdecken zu müssen. Diese Vorsicht war geboten, da nicht auszuschließen gewesen ist, dass aus dem Bieterkollegen bei der nächsten Ausschreibung der Konkurrent wird. Die notwendigen Unterschriften wurden dann in einem Art Gästebuchverfahren eingeholt, bei dem bei einem der Träger das Angebot ausgelegt wurde und die zeichnungsberechtigten Trägervertreter im Laufe von 48 Stunden anreisen und unterschreiben mussten, damit das Angebot bei den bis heute zum Teil unverschämt kurzen Angebotsfristen rechtzeitig zugestellt werden konnte. Immerhin sind hierbei in vielen Regionen Verbünde von Trägern entstanden, die ohne diese Not nur schwer zusammengekommen wären. 
Die kurzen Angebotsfristen führten und führen immer wieder dazu, dass an dem letzten Tag der Angebotsfristen das Reiseaufkommen nach Nürnberg und zu den fünf regionalen Einkaufszentren (REZ) der Bundesagentur rasant zunimmt, da nur wenige Anbieter ihre Angebote den Postdienstleistern anvertrauen. Oftmals wird zudem noch bis in die Nacht vor Ablauf der Angebotsfrist so lange am Angebot gefeilt, dass selbst der beste Expressservice für die wenigen verbleibenden Stunden keine Zustellangebote mehr macht. Wenn dann noch, wie in den Anfangszeiten geschehen, die Öffnungen der Terminbriefkästen an den Abgabestellen für den Umfang der erforderlichen Angebotsunterlagen nicht ausgelegt waren, dann war die Krise perfekt.

Eine andere Auswirkung des neuen Vergabeverfahrens war der Auftragsboom bei Anwaltskanzleien, die sich auf Vergaberecht spezialisiert hatten und die nun mit Mandaten für Nachprüfungsverfahren beauftragt wurden, um zu retten, was noch zu retten war. Für die Geschäftsführer von sozialen und Bildungsträgern, die sich an den Ausschreibungen beteiligten, war es ebenfalls erforderlich, sich umfangreiche Kenntnisse im Vergabe- und Steuerrecht (und hier insbesondere in den bis heute in vielen Fällen noch ungeklärten oder strittigen Fragen der Umsatzsteuerpflicht) und in Fragen des Haftungsrechts zu erwerben. Geht man davon aus, dass auch vorher genug zu tun war, dann konnte diese Mehrarbeit nur durch zusätzlichen internen oder externen Personalaufwand bewältigt werden, natürlich mit den entsprechenden Mehrkosten.

\section{Der tatsächliche Preiswettbewerb}

Da aber mit der Einführung der VOL-gebundenen Vergabeverfahren ein bis heute andauernder und inzwischen ruinöser Preisverfall einherging und geht, mussten die Kosten an anderer Stelle gesenkt werden. Ein Absinken der Preise für Arbeitsmarkt-Dienstleistungen war mit der Verfahrensänderung der Beauftragungen seitens der Bundesanstalt für Arbeit gewollt, insbesondere wollte man die teilweise erheblichen regionalen Preisunterschiede für gleiche Leistungen auf dem unteren Niveau vereinheitlichen.

Das kann aber im Dienstleistungsbereich mit einem Personalkostenanteil von 70 bis 80 Prozent nur zulasten der Löhne und Gehälter gehen. Fast alle gemeinnützigen Organisationen in diesem Arbeitsfeld hatten sich mit ihren Vergütungsregelungen entweder direkt durch Mitgliedschaft in den entsprechenden Tarifgemeinschaften oder indirekt durch angelehnte Haustarife oder Arbeitsverträge an den Tarifen des öffentlichen Dienstes orientiert. Dies war aufgrund des Besserstellungsverbotes im alten Zuwendungsrecht fast schon ein zwingendes Erfordernis.

Daneben gab es aber auch gewerbliche Träger, die andere - in der Regel kostengünstigere - Vergütungsbedingungen in ihren Unternehmen hatten. In einem Vergabewettbewerb, der fast ausschließlich als Preiswettbewerb ausgelegt war, führte dies natürlich zu massiven Problemen bei den gemeinnützigen Trägern. Diese reagierten je nach eigenen Möglichkeiten und Risikobereitschaft mit unterschiedlichen Strategien:
- Die politische Lobby wurde mobilisiert, das Vergabeverfahren abzuschaffen oder zumindest zu modifizieren, um die existenzbedrohenden Auswirkungen zu verhindern. Das war nur zu einem geringen Teil erfolgreich.

- Es wurden Ausgründungen in (gemeinnützige) Gesellschaften mit beschränkter Haftung vorgenommen, in denen deutlich niedrigere Vergütungsstrukturen festgesetzt wurden.

- Andere Träger zogen sich ganz aus diesem Arbeitsbereich zurück.

- Betriebsbedingte Kündigungen waren ebenfalls in vielen Fällen die Folge. Zum einen aus der Notwendigkeit der verlorenen Aufträge heraus, zum anderen um einen Neuanfang mit Neueinstellung der Fachkräfte zu anderen Bedingungen zu ermöglichen.

Wir mussten nach verlorener Ausschreibung in einer anzeigenpflichtigen sogenannten Massenentlassung 1997 mehr als dreißig Mitarbeitern kündigen. Unternehmen, die nicht tarifgebunden waren, versuchten und versuchen mit ihren Betriebsräten oder Personalvertretungen flexiblere aber auch niedrigere betriebliche Vergütungsstrukturen $\mathrm{zu}$ vereinbaren.

\section{Die Abwärtsspirale bei den Gehältern}

In der Folge sanken und sinken die Gehälter von Ausbildern, Dozenten und Sozialpädagogen stetig. Einheitsgehälter (unabhängig von Lebensalter, Berufserfahrung und Zusatzqualifikationen) deutlich unter 2.000 Euro sind auch in den westlichen Bundesländern inzwischen keine Seltenheit mehr. Diese Entwicklung hat auch Einzug in die Fernsehberichterstattung gehalten (WDR-Fernsehen, Westpol, 20.05.2007).

Die Abwärtsspirale der Einkommen der pädagogischen Fachkräfte im Bereich der beruflichen Bildung scheint noch kein Ende gefunden zu haben. Es herrschen wegen fehlender wirksamer tarifvertraglicher Bindungen Zustände wie zu Zeiten der frühen Industrialisierung, wo der Preis (Lohn) der Arbeitskraft einzig über das Angebot und die Nachfrage gebildet wurde. Da im öffentlichen Bereich die Arbeitsplatzangebote für Sozialpädagogen und Sozialarbeiter aufgrund von Haushaltskürzungen eher sinken, aber weiterhin neue Hochschulabsolventen auf den Arbeitsmarkt drängen, sind niedrige Gehälter die Folge. Hinzu kommt, dass die anstellenden Träger aufgrund der Befristung der Aufträge und der Unsicherheit der Folgebeauftragung durch das beschriebene Vergabeverfahren in der Regel nur befristete Arbeitsverträge anbieten (können).

Um guten Mitarbeitern dennoch längerfristige Perspektiven bieten zu können, sind die Träger gezwungen, in der arbeitsrechtlichen Grauzone mit Projektverträgen zu operieren. Die Mitarbeitereinsatzplanung ist dann häufig weniger von einer Passgenauigkeit zwischen Stellenanforderung und Mitarbeiterprofil sondern mehr von Beginn, Ende und Dauer von Projektaufträgen bestimmt. 
Dass unter solchen Bedingungen die Qualität der Projektdurchführung und die Arbeitszufriedenheit der Fachkräfte leiden, muss nicht extra erwähnt werden. Fachkräfte in der Automobilproduktion auf einen Modellwechsel einzustellen, ist eben einfacher, als pädagogische Fachkräfte, die bisher jugendliche Auszubildende sozialpädagogisch unterstützt haben, von einem Tag auf den anderen bei der vermittlungsunterstützenden, psychosozialen Stabilisierung von Langzeitarbeitslosen einzusetzen.

\section{Der Versuch, aus einem Preis- einen Qualitätswettbe- werb zu machen}

Bereits seit mehreren Jahren werden die Vergabeentscheidungen der ausschreibenden Stellen - seit der SGBII-Einführung im Jahr 2005 gehören hierzu zunehmend die Arbeitsgemeinschaften und die optierenden Kommunen - nach der erweiterten Richtwertmethode der UfAB (Unterlage für die Ausschreibung und Bewertung von ITLeistungen - sic!) durchgeführt. Dabei wird in einem ausdifferenzierten Verfahren eine zunächst getrennte Bewertung der inhaltlichen Angebote (Konzept) vorgenommen. Anschließend wird unter Einbezug des Angebotspreises das wirtschaftlichste Angebot ermittelt.

Aber auch hier steckt der Teufel im Detail. Die Ausdifferenzierung der Angebotsbewertung findet häufig doch eher grob statt, so dass es zumeist dann doch das billige (preiswerte) Angebot ist, das den Zuschlag erhält. Außerdem kann ein Angebotskonzept nur hoffen lassen, dass die nach Zuschlag ausgeführte Dienstleistung demselbigen entspricht. Gute Texte bedeuten eben nicht zwangsläufig gute Arbeit. Die Erstellung und Gestaltung der Angebotskonzepte entscheidet damit neben dem Angebotspreis über Erfolg oder Misserfolg. Damit wurde sowohl ein Markt für die Schulung von trägereigenen Angebotsschreibern geschaffen als auch für die externe Erstellung von Angebotskonzepten. Da die Bewertung der Angebotskonzepte von örtlichen Mitarbeitern der Arbeitsagenturen, ARGEn und optierenden Kommunen vorgenommen wird und diese häufig wechseln oder nicht aus dem ausschreibenden Fachbereich kommen, bleibt auch die lesergerechte Gestaltung der Konzepte ein Vabanquespiel.

Um der an vielen Orten beklagten und auch von den Regionalen Einkaufszentren (REZen) der Bundesagentur für Arbeit konzedierten Diskrepanz zwischen der Angebotsqualität (Texte) und der Durchführungsqualität (Praxis) zu begegnen, wurde seitens der Bundesagentur mit den »Handlungsempfehlungen/Geschäftsanweisungen (HEGA) 04/07 - 05 « die »Einführung eines hauptamtlichen Prüfdienstes Arbeitsmarktdienstleistungen « vorgenommen.

Angesiedelt jeweils an den REZen Berlin, Düsseldorf und Nürnberg reisen jetzt diese Prüfteams durch ihre Lande und kontrollieren mehr oder weniger unangemeldet die Durchführungspraxis der Aufträge bei den Trägern vor Ort. Abweichungen zwischen Angebot und Praxis werden sanktioniert. Die Sanktionen reichen von Nachbesserungsverpflichtungen bis hin zu Vertragsstrafen und auch Vertragskündigungen. Leider findet (bisher) eine Berück- sichtigung positiver Prüfergebnisse bei zukünftigen Vergabeentscheidungen nicht statt. Daher bleibt abzuwarten, ob die Arbeit der Prüfteams tatsächlich zu mehr Übereinstimmung zwischen Angebots- und Durchführungsqualität führt.

\section{Die Hoffnung auf Besserung}

Die fehlenden Planungsmöglichkeiten wegen der kurzen Auftragszeiträume, die geringen konzeptionellen und gestalterischen Freiräume wegen der vergaberechtlichen Vorgaben und die schwierigen Arbeitsbedingungen für die Mitarbeiter wegen des Preiskampfes als Herausforderung für die Geschäftsführungen von sozialen Qualifizierungsträgern zu beschreiben, wäre Schönfärberei.

Es zeichnet sich noch keine grundlegende Veränderung zum Besseren hin ab. Die öffentlichen Auftraggeber wollen das Instrument wettbewerbliche Vergabe bei sozialen Dienstleistungen in immer weitere Bereiche hinein ausdehnen. Was fehlt, sind Regeln, die kontraproduktive Auswüchse verhindern. Hiermit meine ich sowohl die unzureichenden Einkommen der in diesem Bereich engagiert tätigen Fachkräfte, als auch Verfahrensregeln, die eine qualitativ hochwertige pädagogische Arbeit verhindern, wie es die in vielen Vergaben zu kurzen Beauftragungszeiträume sind.

So bleibt nur die Hoffnung, dass wieder mehr die konzeptionell-gestaltenden Fähigkeiten eines Geschäftsführers für den Erfolg eines sozialen Unternehmens im Vordergrund stehen.

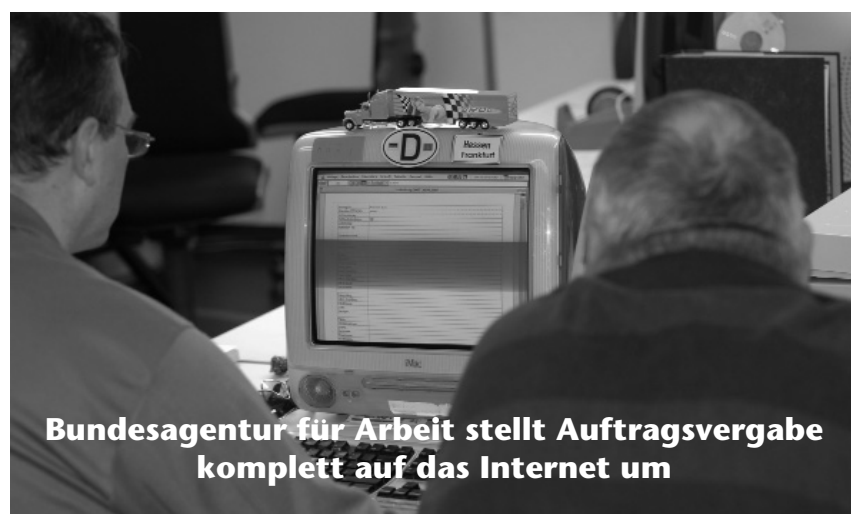

Seit der Novellierung des Vergaberechts im letzten Jahr können öffentliche Auftraggeber die Kommunikationsmittel zur Vergabe öffentlicher Aufträge frei wählen. Die Bundesagentur für Arbeit beabsichtigt daher, voraussichtlich ab dem zweiten Quartal 2008, die Einführung der Vergabe per Internet. Sämtliche Ausschreibungen von Arbeitsmarktdienstleistungen werden dann ausschließlich über die E-Vergabe-Plattform des Beschaffungsamtes des Bundesministeriums des Innern durchgeführt. Zur deren Nutzung muss sich jeder Bewerber registrieren. Die kostenfreie Software »Angebotsassistent AnA» kann nach Registrierung von der Vergabeplattform herunter geladen werden. Das Programm verschlüsselt das Angebot und ermöglicht die elektronische Übersendung an die Angebotsstelle. Die Übermittlung von Angeboten per Post oder durch Boten ist dann nicht mehr zugelassen. An die Stelle der Unterschrift tritt eine elektronische Signatur. Das Beschaffungsamt bietet interessierten Leistungsanbietern Informationsveranstaltungen über die Nutzung der Vergabeplattform an. Die Vergabeplattform ist im Internet unter www.evergabe-online.de erreichbar.

Bundesagentur für Arbeit, 90327 Nürnberg, Telefon 0911 179-0, Fax 0911 1792123, E-Mail Zentrale@arbeitsagentur.de, Internet http://www.arbeitsagentur.de 